\title{
A qualitative exploration of the unique challenges facing older men with haemophilia and the implications for social work practice
}

\author{
Sarah E. Elliott' ${ }^{1}$ Kelsey L. Deane ${ }^{2}$ and Barbara Staniforth ${ }^{2}$
}

\begin{abstract}
INTRODUCTION: For the first time, people who have haemophilia are facing the same aging issues as the general population, adding further complexity to their care and treatment. Worldwide, there has been little research on the psychosocial effects of growing older with haemophilia. This study investigated the holistic experiences of older men with haemophilia in Aotearoa New Zealand. Support services, particularly the roles that social workers could play in facilitating wellbeing, have also been explored.
\end{abstract}

METHODS: A focus group was conducted with a purposive sample of five older men living with haemophilia in Aotearoa New Zealand. Thematic analysis through Braun and Clarke's (2006) six-phased model was used to provide insight into their experiences.

FINDINGS: Substantial physical and new medical challenges existed for the participants and these had flow-on effects for their psychosocial wellbeing. Existing services were well regarded but the anticipated complexity of multi-specialist and continuing care provoked anxiety.

Connection with others with similar challenges was seen as an important social resource.

CONCLUSIONS: The complexities associated with an aging population of people with haemophilia has generated a need for a wide-range of services and supports. Social workers need to take new and different approaches to fill the roles of advocating for, educating, and providing support to older men with haemophilia.

KEYWORDS: haemophilia; ageing; social work; wellbeing
${ }^{1}$ Oranga Tamariki

2 University of Auckland
Haemophilia is a lifelong, genetic condition that causes excessive internal or external bleeding and often results in chronic health problems. Bleeding arises from the lack of an essential blood-clotting factor (World Federation of Hemophilia, 2012). Affected individuals are invariably male, while females, are carriers of the disease but also face different levels of symptoms. Historically, haemophilia contributed to a shorter life expectancy but life expectancy for people with haemophilia is now approaching that of the general male population (Mannucci, Schutgens, Sant'agostino, \& Mauser-Bunschoten, 2009). People with haemophilia are likely to have benefited from improvements in haemophilia care, including the availability of safe, effective blood coagulation treatment (known as factor concentrate, factor, or blood products) which is given intravenously and raises the clotting activity of the blood to a sufficient level to diminish bleeding (Rosendaal et al., 1990).
AOTEAROA

NEW ZEALAND SOCIAL WORK 29(2), 83-95.

CORRESPONDENCE TO: Sarah E. Elliott sarah.preston1@gmail.com 
Other advances include the development of comprehensive care programmes, new approaches to patient management, clinical trials for more effective treatments, and integration of therapeutic modalities (Oldenburg, Dolan, \& Lemm, 2009).

Longer life expectancy for older people with haemophilia brings about new challenges that have never been seen before for the haemophilia population. The physical, mental and emotional effects for those facing haemophilia alongside old age can be substantial (Meijer \& Van der meer, 2007) and add further complexity to their treatment and care. These challenges are exacerbated by significant gaps in expertise and knowledge about the holistic wellbeing of older people with haemophilia.

The majority of the current literature on ageing with haemophilia is framed from a medical perspective, focusing on comorbidities and haematological management; however, a few exceptions exist. Dolan (2010) and Franchini and Mannucci (2010) have focused on the physical wellbeing of older people with haemophilia, particularly in the areas of falls and comorbidities but their research extends to the psychosocial effects that arise from these medical issues. Lambing and Kachalsky (2009) have conducted comprehensive research on older people with haemophilia's physical wellbeing, employment, life satisfaction and complications brought about from losing independence. Focusing more exclusively on psychosocial wellbeing, Mauser-Bunschoten, De Knecht-Van Eekelen, and Smit (2007) found that resilience can be used as a way to address the grief and fear experienced by some older people with haemophilia and Bos (2007) identified that social support could buffer, illness, stress or fear and thus could be used to promote the wellbeing of older people with haemophilia. Importantly, Street, Hill, Sussex, Warner, and Scully (2006) found that haemophilia organisations can play a key role in identifying and implementing psychosocial support and be strong advocates for wellness programmes, practices, and policies to reduce the physical and psychological impacts of ageing. However, one of the most recent studies on older people with haemophilia and psychosocial wellbeing demonstrates that supporting older people with haemophilia can be more complicated than expected. Rolstad (2014) assessed the needs of older people with haemophilia specifically with respect to community-based supports. He found that, despite the need for psychosocial support, the men in his study were reluctant to receive this support and unreceptive to therapeutic interventions.

Although research interest in the novel and complex issues emerging for older people with haemophilia has grown over the last 10 years internationally, few studies have been conducted using the voice of older people with haemophilia themselves. In the Aotearoa New Zealand context, Park and her colleagues (Park, Scott, Benseman, \& Berry, 1995; Park, Scott, \& Bensmen, 1999; Park \& York, 2008) have produced some of the only Aotearoa New Zealand focused research on the haemophilia population. Their anthropological research provides an overview of the experiences and social and demographic characteristics of people with bleeding disorders in Aotearoa New Zealand. While this research gives context to the reality of people with haemophilia in Aotearoa New Zealand, there is not a specific focus on older people with haemophilia. Indeed, all previous research recognises the need for further investigation and new tools, processes, practices and policies to support how professionals work with older people with haemophilia. The current research contributes to the burgeoning evidence base on the holistic wellbeing needs of older people with haemophilia. In doing so, it provides further insights and recommendations with an emphasis on social work practice.

We argue that social workers are a critical part of the professional network people 
with haemophilia rely on for support with respect to their holistic wellbeing. This view is supported by Allen and Kachalsky (2010) who have written one of the few articles which addresses aging with haemophilia and the implications this may have on social work practice with specific regard to the effects that losing independence can have on older people with haemophilia and how social workers may be able to assist in this area. They also highlight that social work with older people with haemophilia is an area that would benefit from more thorough examination. Other contemporary literature that addresses the connection between social work and people with haemophilia focuses primarily on organising insurance for treatment and care (which is not relevant in Aotearoa New Zealand as treatment is publicly funded) and the provision of support to newly diagnosed families, young children, and adolescents (Cassis, 2007). In contrast, during the 1980s, when human immunodeficiency virus (HIV) and hepatitis $\mathrm{C}$ virus (HCV) transmissions increased for people with haemophilia as a result of inadequate blood transfusion screening, the wide-ranging roles played by social workers in supporting people with haemophilia of all ages and their families were more visible in the literature (Allen \& Kachalsky, 2010). These roles included education through workshops and events; supporting people with haemophilia to work through emotional issues and experiences of stigma; planning for the future; connecting people with haemophilia and HIV to others for social support; assisting with practical tasks; and advocating for people's rights. Social workers are equipped with the skills and knowledge to educate, advocate for, empower, and support people with haemophilia (Lauzon, 2008). The complexity of care associated with a growing population of people living longer with haemophilia indicates that there is an increasing need for such wide-ranging support and that a more extensive evidence base, one that can inform social work practice in this area, is needed. Considering the variations in public health policy and practice worldwide, as well as Aotearoa New Zealand's diverse cultural landscape, further inquiry into the experiences of those aging with haemophilia within the local context and how their needs can be met is also essential.

\section{The current study}

To build on initial understandings regarding the experiences of older people with haemophilia in Aotearoa New Zealand, we felt it was important to begin at the source-with those experiencing the challenges, and to use a qualitative approach that could capture the richness of their experiences. The first author worked for the Haemophilia Foundation of New Zealand (HFNZ) as a social worker for seven years. In this role she observed that older people with haemophilia reach "normal" old age only to be faced with additional, but different, challenges to those experienced by people with haemophilia before them. A focus group approach was chosen to further investigate her observations. The specific objectives of this study were to: 1) gather information from the participants about the issues and challenges they faced growing older with haemophilia; and 2) gather opinions of the services and supports available to them. In exploring these objectives we sought to better understand how these men were affected by their condition and the ways in which social workers could offer improved or additional support.

\section{Methods}

A focus group was chosen in order to consult with a select group of aging men affected by haemophilia in Aotearoa New Zealand. Focus groups allow researchers to listen to debate and gain a deeper understanding of participant viewpoints and experiences (Walter, 2006). This focus group study was the first phase of a larger, mixed-method research project. The focus group was also used to pilot a draft questionnaire which would later be distributed to all older people with haemophilia in Aotearoa New Zealand with the aim of capturing the breadth rather than depth of perspectives. As the emphasis of this article is on 
the participants' detailed experiences, we do not include discussion of the feedback participants provided about the questionnaire items. It is important to note that the questionnaire feedback was obtained after the open discussion of their experiences described here.

Ethics approval for the full research project was obtained from the University of Auckland Human Participants Ethics Committee prior to the commencement of this study. All participation was voluntary and participants were asked to respect the privacy of all members and not to disclose or discuss the other participants' identities or information. As the first author was an Outreach Worker for HFNZ at the time this research was conducted, participation in the focus group was sought only from individuals based outside the region where the researcher worked, and the potential conflict of interest was made transparent through the Participant Information Sheet.

\section{Participants}

Focus group participants were identified with assistance from the HFNZ Manager of Outreach Services using purposive sampling by identifying people who met the selection criteria and who represented a variety of ages, ethnicities, bleeding disorder severities, and experiences. Fourteen men met the criteria and were invited to participate in the focus group via posted letter. Five participants, who ranged in age from 45-72 years, agreed to participate in the focus group. One participant identified as Māori, one as Māori/New Zealand (NZ) European, and three as NZ European. Four of the participants had severe haemophilia and one had moderate haemophilia (three different severities exist: mild, moderate and severe). Three participants lived within a one-hour drive of a Haemophilia Treatment Centre (HTC), and the other two lived further away from their HTC.

\section{Procedure}

The focus group was held in a meeting room in August, 2014. A shared dinner was first paid for by HFNZ to acknowledge participants' time and participation. Participants were informed of their rights and subsequently provided written consent to participate and to have the focus group audio-recorded. The focus group discussion was facilitated by the first author and guided by a semi-structured interview schedule including questions to address the dual aims of the research.

The first author took written notes of the participants' responses and organised these by key themes identified in their narratives as the discussion progressed. These themes were then reviewed with the group to ensure the ideas were accurately represented and adjustments made until the themes were correctly captured and reflected the conversation that had taken place. The focus group lasted 90 minutes and each participant was given a koha (\$20 grocery voucher) as a token of appreciation.

\section{Data analysis}

The focus group audio recording was manually transcribed after the workshop and the written transcript was thematically analysed following Braun and Clarke's (2006) six-step methodology where, after researchers became familiar with the data, codes were identified, and these were then reviewed to identify themes. The number of times a particular issue was discussed (by the same or different participants) was also taken into account, as was the time taken to discuss an issue, the emotion generated by an issue, and if an issue was agreed upon, or not, by all participants. The coding and themes were then reviewed by all three authors for internal homogeneity within thematic categories and for external heterogeneity across categories.

\section{Findings}

The findings are presented according to the two research aims, with the first section focusing on the experiences of older people with haemophilia with respect to the challenges and issues they are currently facing and the second focusing on their perceptions of 
the supports and services currently available to them. The overarching themes are represented as section headings and subthemes are italicised for ease of identifiability.

\section{Challenges and issues facing older people with haemophilia}

\section{Physical health}

Participants described physical health, specifically decreased mobility and decreased physical activity as the most prominent issue for them, all agreeing that decreased mobility and physical activity negatively affected their wellbeing in very serious ways. Participants reported that decreased mobility had caused their movement to slow, so they were no longer able to participate in sports and activities they had once enjoyed. Some talked angrily about reoccurring bleeding episodes which had resulted in reduced joint movement and arthritis. They expressed frustration, knowing that exercise was good for them and their joint health, but being unable to participate in activity. For instance, one argued, "I want to get back to walking [on the golf fairway]. I used to do it all the time, but I can't, not even with my walker". They laughed about their "golf swing getting worse and worse" and how much their bodies had deteriorated over time. Participants also worried that they would lose further mobility, as expressed by one participant, "[mobility will] continuously slide away to a point where we are immobile".

\section{New medical problems}

The participants described the ways their bodies were "breaking down" and noted they were experiencing comorbidities, other diseases on top of their haemophilia, which one man described as "all sorts of weird stuff". They explained it was hard enough to deal with haemophilia without the complication of managing new medical problems because of aging. One stated "it's all the other things that now come on top of the challenges that we have already had to face in life".
These new conditions led to complexities for their care and treatment. They indicated that all of their medical needs were once fully managed by the HTCs and that they had rarely engaged with specialists or GPs, but this could no longer be the case with the onset of comorbidities. They also felt that haemophilia treatment once fixed everything for them, however, now they needed additional medications for their new old age conditions. As stated by one participant, "for everything we used to go and see the Haematology Department and get a shot factor, and that was the answer to every problem we ever had". Another stated, "you can't give yourself another shot of factor and fix the heart problem". They recognised that with new medical problems came a higher risk for medical intervention and surgeries which are concerning for older people with haemophilia due to excessive bleeding. One participant explained, "it's those complexities around all the other things; gall stones, heart problems, liver problems ... where they are required to go inside you [operate] to fix it, that's where it gets complicated".

Coping with multiple comorbidities appeared to present many challenges for the participants, particularly as there was not much knowledge or experience of this within the haemophilia community. The group anticipated that things were going to become more difficult for them medically as they continued to age, and they would increasingly need additional specialists involved in their care, along with medications, and surgeries. They talked at length about the challenges comorbidities presented for them in many areas of their lives (mental and emotional, physical, logistical, and financial).

\section{High financial cost of haemophilia to the healthcare system}

Participants worried that the very high cost of their haemophilia treatment to the healthcare system would prevent them from getting the service, care, operations, and treatments they 
would need. They recognised that District Health Boards (DHBs) are "tightening the screws" and constantly analysing the money people with haemophilia cost the system. One participant said "at some stage I would imagine that they are going to start doing the math and doing cost benefit analysis-if we fix this guy's ticker is it worth the cost?" There was nervousness as the participants expressed concern that it would be them, the older people with haemophilia, who would miss out first unless they could strongly "prove their case on financial terms". One participant shared, "I know two cases where people [with haemophilia] have gone to the surgeons and the surgeon says: you need this operation or replacement but we don't have the funds for it". Another participant was denied surgery due to its cost.

Two participants commented that clinicians appeared to be talking increasingly about the cost of their haemophilia treatment in front of them, and some had seen the costs of their treatment displayed on a price list at the blood bank when collecting their treatment. This issue clearly affected their mental and emotional wellbeing, with words such as "nervous," "fear", and "worry" being repeated throughout the dialogue.

\section{Losing independence}

The participants alluded to their fears of losing independence subtly throughout the focus group. They were concerned about the likelihood of having to spend more time in hospital as they got older, due to things such as: longer recovery times, more hospital visits and stays due to comorbidities, and an inability to treat themselves. One participant talked about his negative hospital experiences saying, "I resent going to hospital, I hate hospitals," and they all agreed they would much rather stay in their homes and try to be independent for as long as possible.

Participants mentioned concerns about being able to continue caring for themselves. They worried that, as their eyesight, veins or dexterity deteriorated with age, they would have to rely on medical professionals to administer intravenous treatment instead of being able to self-administer. One participant already experienced this challenge, "I can't do that [treat at home]. I can't give it to myself because I'll miss the vein all the time". This was especially a concern for those with severe haemophilia who needed to treat themselves more regularly (i.e., multiple times a week). They also talked of how more frequent hospital visits would create practical, financial and logistical challenges.

\section{Perception of services and supports for older people with haemophilia}

Participants indicated that they felt "very spoilt" in Aotearoa New Zealand regarding services and supports. Nevertheless, they offered suggestions for further service improvements. The following section presents the detailed findings in relation to this second research objective.

\section{Haemophilia Treatment Centres (HTCs)}

All participants strongly agreed that they had great services, support, and positive experiences with their HTC. They conveyed very high praise for their specialist nurse and spoke fondly of their haematologists, physiotherapists, and surgeons. They compared the medical care they received in Aotearoa New Zealand to developing countries, and were very appreciative of what they have here: "Compared to places like Cambodia we've got brilliant service. You look at the oldest people in Cambodia [with haemophilia] and their life expectancy is around $30^{\prime \prime}$.

They were also grateful for the Aotearoa New Zealand comprehensive care model, with one stating "there are not many gaps" in services, and another saying the comprehensive care team "are committed to really looking after this community; it is really exceptional". Words such as "welloiled machine", "caring" and "genuine" were used repeatedly to describe their 
HTCs. Their access to their medical team impressed them most, especially to nurses. One explained, "you ring [nurse] at any time of the day or night and [nurse] is virtually guaranteed to answer that phone within three rings". Another participant revealed, "when I came out of hospital with a bleed and I'd rung [nurse] and [nurse] came and picked me up and drove me home, how amazing is that?" This also extended to haematologists as expressed by another participant, "it used to be that I could call [haematologist] at home at three in the morning". An orthopaedic surgeon was spoken about fondly by another, "you don't even make an appointment, just turn up and I will see you now". They also spoke warmly of their haemophilia physiotherapist and group physiotherapy sessions and initiatives.

Appreciation of medical staff was closely connected to another key theme: their fear and concern that HTC staff would leave their jobs, leaving gaps in services and people without specialist skills and knowledge. One participant stated, "if you take one of those things out of the equation ... then we lose our point of contact". Participants described that they "rely on" and "need" their nurses and specialists involved closely in their care. They used words such as; "fear," "nervousness," and "risk" frequently when discussing the impact of medical staff leaving, or positions being cut back. In summing up their feelings on this topic, one shared, "you live in fear that one of them will leave," and another expressed, "I'm always really nervous that they are going to pull the plug on it [physiotherapy service] or they will just quit and move on".

\section{Complexity of multiple specialists}

There was some concern expressed about decentralised services because comorbidities and new medical problems required the involvement of other medical professionals. This presented problems as the participants said that new medical professionals often lacked understanding about haemophilia and its complexities. One man shared his experience, saying he had told his GP about having haemophilia and his GP said, "oh I don't know anything about that". They all commented on having to educate medical professionals about their condition and its implications. One participant had to do so at the emergency department at the age of eight. They were also worried about poor communication between specialists, GPs, and their HTCs.

The group discussed at length the physical and practical implications of needing multiple specialists located in different settings, whereas it "used to be a one stop shopyou'd just go to the hospital for everything". With services no longer all in one setting, there would likely be difficulties with traveling, parking, stress and problems for people with mobility issues or who lived in isolated places. One participant reflected, "it's hard if you are having mobility problems and you've got to travel all around the countryside to get the specialists". They were particularly worried for others with haemophilia who lived outside of the main centres of Aotearoa New Zealand and their access to quality care: "We hear horrible stories of guys in outlying areas that have been virtually forced to move to the cities because they just can't get the level of service or care".

\section{Social connection}

Participants mentioned the importance of bonding and connecting with other people who have haemophilia. They also mentioned the importance of their comradery and their ability to understand one another, claiming it is often the "bonding and friendship" that helped them through the tough times. They worried about those who were not connected to others with haemophilia, expressing concern for the "bunch of guys isolated out there on their own, doing their own thing, wondering why the world hates them". However, the group recognised that HFNZ was "probably scratching their head" on how to best support the older people with haemophilia. Some felt HFNZ was not supplying the types of events that 
benefited and suited older men, and that HFNZ may not understand how older men communicated and engaged with one another. Participants' ideas included events specifically targeted to men's interests, and doing activities alongside one another. One suggested "give us a beer and a fishing rod and we'll talk the day away". Other than suggestions for specific types of events and ways of engaging them, four out of five of the participants generally believed HFNZ was supportive of them and their needs. One claimed it was the "best support we've ever had, and they are always looking at improving," and another said "services are bang on as far as I am concerned".

\section{Discussion}

This study aimed to explore both the concerns and challenges facing older people with haemophilia and their perceptions of existing supports and services. It was our intention to keep the questions broad and to let the participants guide the discussion based on what was important to them at that point in time. In capturing what was most salient to them with respect to these topics, we were able to identify: 1 ) if they saw social workers as a key and positive support in their lives, and 2) how social workers could play a stronger role in supporting their quality of life.

We discovered that the participants' concerns were predominantly focused on physical health and medical issues and the participants affirmed the importance of having coordinated medical services to address both haemophilia and other medically related aging issues arising from the complexity of managing both concurrently. Nevertheless, they articulated a clear connection between the physical challenges and their psychosocial wellbeing and pointed to the importance of recognising the link between their need for extensive medical care and its wide-ranging impact on their lives. Our findings intimate that greater education on the complexity of issues faced by older people with haemophilia and advocacy for retaining well-connected, comprehensive services that address the holistic needs of older people with haemophilia are required. Social workers are well-suited to these roles as evidenced by their wide-ranging involvement in the $\mathrm{HIV}$ and HCV transmission scares in the 1980s (Allen \& Kachalsky, 2010) and their role in supporting and assisting children and young adults with haemophilia and their wider whānau with accepting and living well with their condition through education, advocacy and capacity building (Cassis, 2007). Haemophilia social workers can also draw on their experience of providing psychoeducation to families, and in identifying the possibilities and resources that can be accessed in various systems and communities. Findings and implications for social work practice are now explored in further detail.

\section{Concerns and challenges expressed by older people with haemophilia}

The most prevalent, serious, and frequently reported-upon issues in this study were the loss of mobility and decreasing physical ability. These presented challenges and very real fears for participants. They worried that in the future they would become completely immobile. This is a common finding for older people with haemophilia around the world, and one that can have a serious effect on quality of life (Chen et al., 2015). Comorbidities also presented a number of challenges and future worries for older people with haemophilia in many areas of their lives (mental and emotional, physical, logistical, and financial). Existing evidence clearly demonstrates that comorbidities are becoming more common in older people with haemophilia and they are becoming more challenging for healthcare providers and patients to manage (Franchini \& Mannucci, 2010).

This group of older people with haemophilia expressed growing fear that they would no longer be able to give themselves their own haemophilia factor 
treatment. This would create further medical and practical challenges due to more frequent (in some cases daily) hospital visits or choosing not to have treatments (which could result in more bleeds, pain and decreased mobility). Accompanied by the new and very real possibility of family members (i.e., spouse) passing away before the older people with haemophilia that they support, adds further complexity to this issue of treatment at home, as it is often family who help with giving treatment when the older people with haemophilia cannot do so themselves. Lambing and Kachalsky (2009) explored the issue of the increasing need for care outside the home and the effects that no longer being able to self-treat could have on a person's life. They also found the need for more attention, education and resources to help older people with haemophilia find ways to maintain independence and deal with the physical and psychological consequences of losing independence. This was an issue rarely explored elsewhere and one that will become more prevalent over time.

Haemophilia treatment costs the Aotearoa New Zealand healthcare system approximately $\$ 25$ million per annum (Pharmac, 2015), and our participants expressed fears that the high cost of their haemophilia treatment could prevent them from getting the services, care and operations they may need in the future. This could also lead to older people with haemophilia reducing treatments to save money (as is happening with one participant) or experiencing immense feelings of guilt. This was a concern discussed in research with Park (Park et al., 1999; Park \& York, 2008) with PWH of mixed ages in Aotearoa New Zealand. Park and colleagues also found that their participants were internalising how expensive they were to the healthcare system. There was little mention of this issue in the international literature which suggests that it may be specific to Aotearoa New Zealanders or that it has not yet been made visible overseas. Further intentional investigation is thus warranted.

\section{Important social supports and services for older people with haemophilia}

The older people with haemophilia in this study highly valued social participation, connection, and support from other people with bleeding disorders. However people with haemophilia in general may have problems with social participation due to time in hospital and physical inability to participate or attend events/functions (Triemstra et al., 1998); wanting to hide their condition due to discrimination and stigma (Barlow, Stapley, Ellard, \& Gilchrist, 2007); or mental health barriers such as stress, high anxiety, low self-esteem, and depression (Ghanizadeh \& Baligh-Jahromi, 2009). Considering that Bos (2007) found social support can buffer the ill-effects of illness and stress for older people with haemophilia, strategies are needed to help older people with haemophilia overcome barriers to social participation. In this regard, the current findings that HFNZ and its social workers do not always meet the needs of this group of people in terms of the events and activities on offer is of concern. Nevertheless, it is not unusual, as haemophilia organisations internationally struggle with how to involve older people with haemophilia in age- and gender-appropriate activities at appropriate times and places (Rolstad, 2014). Street et al. (2006) also comment that it is hard for haemophilia organisations to meet the needs of older people with haemophilia when their needs are unknown.

The participants in this study also encountered a lack of knowledge about haemophilia in the community and among many medical professionals. older people with haemophilia are finding they need to educate professionals and retell their stories and experiences over and over again. Education and advocacy could make a difference in these regards. Social workers are often involved in care coordination and case management of both older people and people with chronic conditions. Using a comprehensive care model may significantly increase the quality, efficiency and healthrelated outcomes of care (Boult et al., 2009). 


\section{The implications of these findings for social work practice}

Thre are a number of areas where social workers could play key roles to improve the lives of older people with haemophilia. Social workers often recognise the resilience of the people that they work with, and older people with haemophilia are an excellent example of people who have survived, or even thrived, with a complicated illness. older people with haemophilia are also forging new territory as they live longer than people with haemophilia ever have. Solution-focused (Corcoran \& Pillai, 2009), or strengths-based (Nelson-Becker, Chapin, \& Fast, 2013) approaches should be used first and foremost to explore older people with haemophilia's skills and empower them in coming up with new ways of coping.

Some older people with haemophilia may require or benefit from support to address and work through their multiple fears and anxieties, including their loss of independence, mobility and the increased guilt they feel about the cost of their haemophilia treatment. Although it was found that multiple fears exist, MauserBunschoten et al. (2007) found that older people with haemophilia also have very high resilience and optimistic views. Social workers therefore could help to strengthen their existing resilience and coping mechanisms, in addition to connecting older people with haemophilia to a range of therapeutic support options.

Social workers should help older people with haemophilia plan and prepare for their future and engage in greater advocacy towards meeting their needs. This could be done by organising and coordinating treatment for those who can no longer treat themselves, through discussions with local GPs or outreach nurses or facilitating whānau or professional meetings to see who could take on required tasks. Social workers can also facilitate access to the correct supports and equipment for mobility needs or assist in advocating for further investment in life-saving haemophilia treatments and operations (through the public and private sphere) that could drastically impact the quality of life for older people with haemophilia.

A leading role in connecting older people with haemophilia to one another could be facilitated by social workers. This is particularly important for those who live in isolated locations. This connection could help reduce some of the psychological impacts of aging by sharing, connecting, and increasing older people with haemophilia's understanding that they are not alone. This could be aided by ensuring social workers have accurate and current records on the population concerned to guarantee all older people with haemophilia are getting upto-date information, support, invitations to events, and outreach visits. Through such connection, social workers could also gain better information about older people with haemophilia's needs and encourage these men to participate in the planning and development of much-needed wellness programmes. In discussions with older people with haemophilia, social workers can gather information to inform HFNZ what events appear relevant, appropriate and engaging for older people with haemophilia. They can also ensure practices, policies, and supports are relevant and directed at preventative strategies to reduce the physical and psychological impacts of aging.

Social workers should play a key role in upskilling and educating GPs and other medical professionals. They can assist in explaining the medical condition, providing educational resources and also helping medical professionals understand the psychosocial impacts of haemophilia. They could attend appointments alongside older people with haemophilia or help them to liaise with their medical health professionals. Social workers could also play a role in educating medical professionals about how they could best connect with older people with haemophilia, for instance by highlighting the need to be more careful and sensitive in their discussions, including 
being mindful about their tone and language when discussing the cost of treatments and surgeries for older people with haemophilia so as not to provoke further anxiety.

\section{Study limitations and future research directions}

Evident limitations of the study include its small scope (one focus group from one geographic location), and that individuals who had low mood, pain, mobility issues, or who were in hospital, might have been less likely to be interested in or able to attend the focus group. Thus, the results are not generalisable and are possibly biased in a positive direction.

In addition, although our open approach to the questioning with limited prompting allowed the participants to express what was most relevant to them, the predominant focus was on both challenges and services of a medical nature, which provided very little insight into their holistic wellbeing. Even when supports were mentioned, participants primarily took it to mean the medical team around them, along with some social support from people in the haemophilia community. There was no discussion at all of cultural or spiritual support, support from family or friends, or wider (non-haemophilia focused) social support, which we had expected, especially given the importance many tangata whenua place on the spiritual and relational dimensions of wellbeing (Durie, 1985) and the involvement of Māori participants in our study. It is unclear whether this was due to their medical issues actually being at the forefront (or root cause) of their daily life experiences to the point of obscuring other dimensions of their wellbeing or if they were perhaps reluctant to describe psychosocial, emotional and spiritual challenges openly in front of their peers. They may also have been more guarded because the interviewer was employed by HFNZ. Given the potential for social workers to provide wide-ranging support, as described above, this also raises concerns that social work may not currently have enough visibility in the lives of older people with haemophilia.

The questionnaire study conducted as a follow-up to this initial qualitative exploration focused more explicitly on the multiple dimensions of wellbeing, including spirituality and family relationships and was designed to address some of these limitations. These findings will be reported in future articles. Further research should also consider directly comparing the experiences of older people with haemophilia to those of older people with other chronic conditions or disabilities and the general aging population. Our findings indicate they are likely to face similar challenges such as carrying out daily self-care activities, living independently, carrying out essential social roles, increased risk of hospitalisation, and physical inactivity and functional limitations (Heikkinen, 2006). Nevertheless, different conditions are also likely to give rise to unique challenges requiring tailored social work support coordinated with specialists such as gerontologists, local aging organisations (e.g., Age Concern), aging experts, and others involved with positive aging.

\section{Conclusion}

To date, there has been limited exploration into the unique issues faced by older people with haemophilia, despite consensus that assessment of the needs of older people with haemophilia is urgently required (Franchini \& Mannucci, 2010). This research adds to the assessment of needs by clearly presenting some of the major challenges faced by older people with haemophilia and describing their perceptions regarding the support they receive. Social workers appear well situated with expertise and experience to either adapt or create new practices, and interventions to address the needs of older people with haemophilia. This study reiterated findings from previous studies around physical ability and immobility and the need for further education, advocacy and practical support for older people with
ACKNOWLEDGEMENTS: HFNZ contributed to this research by providing support with recruitment and partial funding. We are also grateful for the contribution made by the participants. 
haemophilia. This study also described lesser-known concerns associated with losing independence and no longer being able to self-treat, the emotional burden felt in relation to the cost of haemophilia treatment, and the desire for social connection with other older people with haemophilia. These findings help to deepen understanding of the lives and perceptions of older people with haemophilia in Aotearoa New Zealand. Through this research social workers, other haemophilia professionals and the wider haemophilia community are provided with knowledge to improve their policies, practice, programs and service to enhance the lives and wellbeing of older people with haemophilia.

\section{References}

Allen, K., \& Kachalsky, E. (2010). Aging with hemophilia: Implications for social work practice. Social Work in Health Care, 49(4), 327-344. doi:10.1080/00981380903520434

Barlow, J., Stapley, J., Ellard, D., \& Gilchrist, M. (2007). Information and self-management needs of people living with bleeding disorders: A survey. Haemophilia, 13(3), 264-270.

Bos, R. (2007). As haemophilia patients are aging... In E. Mauser-Bunschoten, A. De Knecht-Van Eekelen, \& C. Smit (Eds.). Aging with haemophilia: Medical and psychological impact (pp. 87-93). Utrecht, Netherlands: Van Creveldkliniek-Haemotology.

Boult, C., Green, A. F., Boult, L. B., Pacala, J. T., Snyder, C. \& Leff, B. (2009). Successful models of comprehensive care for older adults with chronic conditions: Evidence for the Institute of Medicine's "retooling for an aging America" report. Journal of the American Geriatrics Society, 57(12), 2328-2337.

Braun, V., \& Clarke, V. (2006). Using thematic analysis in psychology. Qualitative Research in Psychology, 3(2), 77-101.

Cassis, F. (2007). Psychosocial care for people with Hemophilia. Montreal, Canada: World Federation of Hemophilia.

Chen, C., Huang, K., Chen, C., Huang, S., Huang, C., Chen, Y., \& Hsu, S. (2015). The impact of joint range of motion limitations on health-related quality of life in patients with haemophilia A: A prospective study. Haemophilia, 21(3), 176-184.

Corcoran, J., \& Pillai, V. (2009). A review of the research on solution-focused therapy. British Journal of Social Work, 39(2), 234-242.

Dolan, G. (2010). The challenge of an ageing haemophilic population. Haemophilia, 16(5), 11-16.

Durie, M. (1985). A Māori perspective of health. Social Science \& Medicine, 20(5), 483-486.

Franchini, M., \& Mannucci, P. M. (2010). Co-morbidities and quality of life in elderly persons with haemophilia. British
Journal of Haematology, 148(4), 522-533. doi:10.1111/ j.1365-2141.2009.08005.x

Ghanizadeh, A., \& Baligh-Jahromi, P. (2009). Depression, anxiety and suicidal behaviour in children and adolescents with Haemophilia. Haemophilia, 15(2), 528-532.

Heikkinen, E. (2006). Disability and physical activity in late life-Research models and approaches. European Review of Aging and Physical Activity, 3(1), 3-9.

Lambing, A., \& Kachalsky, E. (2009). The new age of haemophilia. Haemophilia, 15(6), 1330-1331. doi:doi. org/10.1111/j.1365-2516.2009.02076.x

Lauzon, C. (2008). Still standing. Waiuku, New Zealand: W. J. Deed Printing.

Mannucci, P. M., Schutgens, R. E., Sant'agostino, E., \& Mauser-Bunschoten, E. P. (2009). How I treat agerelated morbidities in elderly persons with hemophilia. Blood, 114(26), 5256-5263.

Mauser-Bunschoten, E., De Knecht-Van Eekelen, A. \& Smit, C. (2007). Aging with haemophilia: Medical and psychological impact. Utrecht, Netherlands: Van Creveldkliniek-Haemotology.

Meijer, K., \& Van der meer, J. (2007). Invasive diagnostics and surgical interventions. In E. Mauser-Bunschoten, A. De Knecht-Van Eekelen, \& C. Smit (Eds.), Aging with haemophilia: Medical and psychological impact (pp. 69-76). Utrecht, Netherlands: Van CreveldkliniekHaemotology.

Nelson-Becker, H., Chapin, R., \& Fast, B. (2013). The strengths model with older adults: Critical practice components. In D. Saleebey (Ed.), The strengths perspective in social work practice (6th ed., pp. 161-181). Boston, MA: Pearson.

Oldenburg, J., Dolan, G., \& Lemm, G. (2009). Haemophilia care then, now and in the future. Haemophilia, 15, 2-7. doi:10.1111/j.1365-2516.2008.01946.x

Park, J., Scott, K., \& Benseman, J. (1999). Dealing with a bleeding nuisance: A study of haemophilia care in New Zealand. The New Zealand Medical Journal, 112(1087), 155-158.

Park, J., Scott, K., Benseman, J., \& Berry, E. (1995). A bleeding nuisance: Living with haemophilia in Aotearoa New Zealand. Auckland, NZ: Department of Anthropology, The University of Auckland.

Park, J., \& York, D. (2008). Social ecology of new technologies and haemophilia in NZ-A bleeding nuisance revisited. Auckland, NZ: Department of Anthropology, The University of Auckland.

Pharmac. (2015). Inpharmation-August 2015. Retrieved from https://www.pharmac.govt.nz/newsletters/ inpharmation/inpharmation-2015-08/

Rolstad, E. (2014). Perceptions of men with moderate to severe hemophilia regarding the management of their chronic disorder and utilization of community-based support. American Journal of Men's Health, 9(6), 486-495. doi:10.1177/1557988314551360

Rosendaal, F. R., Smit, C., Varekamp, I., Brockervriends, A., Van Dijck, H., Suurmeijer, T. M., ... Briët, E. (1990). Modern haemophilia treatment: Medical improvements and quality of life. Journal of Internal Medicine, 228(6), 633-640. 
Street, A., Hill, K., Sussex, B., Warner, M., \& Scully, M. F. (2006). Haemophilia and ageing. Haemophilia, 12, 8-12. doi:10.1111/j.1365-2516.2006.01254.x

Triemstra, A., Van der Ploeg, H., Smit, C., Briet, E., Ader, H., \& Rosendaal, F. (1998). Well-being of haemophilia patients: A model for direct and indirect effects of medical parameters on the physical and psychosocial functioning. Social Science \& Medicine, 47(5), 581-593.

Walter, M. (2006). Social research methods. Oxford, UK: Oxford University Press.

World Federation of Hemophilia. (2012). What is hemophilia? Retrieved from http://www.wfh.org/en/page.aspx?pid=646 